Jagpreet Singha, Sukhmeen Kaura, Gaganpreet Kaur, Soumen Basu and Mohit Rawat*

\title{
Biogenic ZnO nanoparticles: a study of blueshift of optical band gap and photocatalytic degradation of reactive yellow 186 dye under direct sunlight
}

https://doi.org/10.1515/gps-2018-0084

Received April 19, 2018; accepted July 20, 2018; previously published online August 31, 2018

Abstract: Synthesis of nanoparticles (NPs) using plant extracts has been suggested as an environmentally friendly alternative to chemical synthesis of semiconductor NPs. In the present study, ZnO NPs were synthesized by a simple and cost-effective method using Coriandrum sativum leaf extract and zinc acetate as precursors. The as-synthesized ZnO NPs were characterized by UV-visible spectroscopy, transmission electron microscopy (TEM), Fourier transform infrared (FTIR) spectroscopy, X-ray diffraction (XRD), field emission scanning electron microscopy (FESEM) and energy dispersive X-ray (EDX) analysis. The results confirmed the formation of $\mathrm{ZnO}$ NPs with a wurtzite structure, spherical shape and average particle size of $24 \mathrm{~nm}$. The photocatalytic degradation of reactive yellow 186 (RY 186) dye was carried out under direct sunlight irradiation and its degradation efficiency and apparent rate constant $\left(\mathrm{K}_{\text {app }}{ }\right)$ of reaction were calculated to be $93.38 \%$, and $0.0019 \mathrm{~min}^{-1}$, respectively. The optical band gap value of the as-synthesized $\mathrm{ZnO}$ NPs was found to be $3.4 \mathrm{eV}$, which indicates the presence of blueshift. Owing to the presence of blueshift and a wide band gap of synthesized biogenic ZnO NPs, the overall absorption of sunlight irradiation will be enhanced, which leads to higher degradation efficiency of the dye. The current study thus highlights the optical band gap properties of biogenic $\mathrm{ZnO}$ NPs and their significance as a heterogeneous catalyst for the purification of polluted water.

\footnotetext{
Jagpreet Singh and Sukhmeen Kaur: These co-authors equally contributed as first authors.

*Corresponding author: Mohit Rawat, Department of Nanotechnology, Sri Guru Granth Sahib World University, Fatehgarh Sahib-140406, Punjab, India, e-mail: mohitnano.nit@gmail.com Jagpreet Singh and Sukhmeen Kaur: Department of Nanotechnology, Sri Guru Granth Sahib World University, Fatehgarh Sahib-140406, Punjab, India

Gaganpreet Kaur: Department of Computer Science and Engineering, Sri Guru Granth Sahib World University, Fatehgarh Sahib-140406, Punjab, India

Soumen Basu: Department of Chemistry and Biochemistry, Thapar University, Patiala-147004, Punjab, India
}

Ә Open Access. (c) BY 2019 Walter de Gruyter GmbH, Berlin/Boston
Keywords: blueshift; Coriandrum sativum; green synthesis; optical band gap; photodegradation; reactive yellow 186; ZnO NPs.

\section{Introduction}

Semiconductor nanoparticles (NPs) exhibit exceptional optical, electrical, chemical and magnetic properties as compared to bulk materials. Owing to the peculiar properties of metal oxide NPs, they have attracted much interest from both fundamental and technological researchers. $\mathrm{ZnO}$ is a semiconductor material with a wide band gap of about $3.3 \mathrm{eV}$ and high excitation binding energy of about $60 \mathrm{meV}$ [1]. Due to their band gap and unique optical properties, ZnO NPs have gained a lot of attention in the fields of UV absorption, antibacterial treatment, UV light emitters, photocatalysts, solar cells, gas sensors, luminescent materials, bioimaging and drug-delivery [2-5].

Dyes with synthetic origins and complex structures are difficult to degrade and hazardous for the environment; it is therefore necessary to remove such dyes from the environment $[6,7]$. A variety of traditional techniques with semiconductor photocatalysts which are presently used for the removal of colored effluents in industrial water are not accurate and do not lead to complete removal of the dyes, because dyes are highly soluble and are resistant to chemical and biological degradation [8]. $\mathrm{ZnO}$ is considered to be a very good photocatalyst, because it is a strong oxidizing agent, less expensive, non-toxic and has high photostability $[4,9]$.

ZnO NPs can be synthesized by various chemical and physical routes including the hydrothermal method, the sol-gel method, the laser ablation method, the sonochemical technique, the mechanochemical method and the microwave technique [10-14]. However, some of these methods require high pressure and high temperature, while others require inert atmosphere and toxic chemicals [15]. In addition, these routes include complex and expensive synthesis and purification processes making them less preferable.

Recently, a biological synthesis of $\mathrm{ZnO}$ NPs via microorganisms and plant extracts has been developed as an alternative to chemical and physical routes due to its 


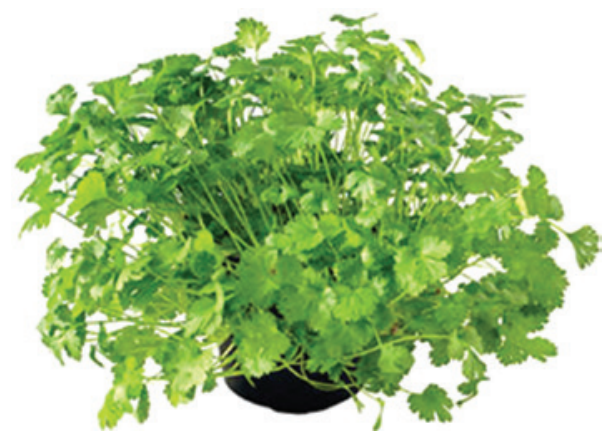

Figure 1: Coriandrum sativum leaves.

cost-effectiveness, simple handling, nontoxicity and ecofriendly nature [16, 17]. Until now, ZnO NPS have been synthesized by using various plants such Cassia Auriculata flower, Azadirachta indica, Parthenium hysterophorus, Calotropis procera latex, Aloe barbadensis miller leaf and grapefruit [3, 18-21].

Among various materials, $\mathrm{ZnO}$ NPs exhibit appreciable applications for environmental remediation applications in light of their benign nature and facile synthesis. To this end, the biogenic $\mathrm{ZnO}$ NPs synthesized using leaf extract of Coriandrum sativum were utilized as the photocatalyst for the removal of reactive yellow 186 (RY 186) industrial dye from water. Coriandrum sativum is a type of herb from the Apiaceae family that includes essential oils and fatty oils and has antimicrobial, anti-oxidant and anti-cancer properties (Figure 1). We also investigated the blueshift of the optical band gap of synthesized biogenic ZnO NPs and total dye removal efficiency with kinetic parameters. To the best of our knowledge, this is the first study to use biogenic ZnO NPs as a photocatalyst for the removal of RY 186 dyes from water. Also, the attractiveness of our study is the utilization of sunlight irradiation instead of artificial light sources (UV lamp, tungsten bulb etc.)

\section{Materials and methods}

\subsection{Materials used}

Coriandrum sativum leaves were bought from fruit market. Fatehgarh Sahib, Punjab, India. Zinc chloride (99\% pure) was purchased from Sigma Aldrich, India and used as a chemical reagent. Distilled water was used throughout the experiment. All reagents used in this experiment were of high purity grade.

\subsection{Preparation of Coriandrum sativum leaf extract}

Coriandrum sativum leaves were collected from nearby gardens, washed very carefully to remove the dust particles and dried in sunlight. Afterwards, the leaves were crushed into a fine powder using a mortar and pestle. Then, $10 \mathrm{~g}$ Coriandrum sativum leaf powder was dissolved in $100 \mathrm{ml}$ of distilled water and stirred at $100^{\circ} \mathrm{C}$ for $15 \mathrm{~min}$. The solution was then filtered with 1.5 micron Whatman filter paper No: 1. The stepwise procedure for the synthesis of ZnO NPs is described in Figure 2.

\subsection{Synthesis of NPs}

Zinc oxide NPs were synthesized using zinc acetate as a salt and prepared plant extract. Briefly, $0.21 \mathrm{M}$ zinc acetate solution was prepared in DI water. After that, $40 \mathrm{ml}$ of Coriandrum sativum leaf extract was added dropwise to this solution and stirred for $4 \mathrm{~h}$ at $70^{\circ} \mathrm{C}$. Then, it was kept on a hot plate for $1 \mathrm{~h}$. Lastly, the solution was calcinated for $4 \mathrm{~h}$ at $400^{\circ} \mathrm{C}$ leaving white colored ZnO NPs.

\subsection{Photocatalytic activity of ZnO NPs}

The photocatalytic potential of the as-prepared NPs was investigated by the degradation of RY 186 dye under sunlight $\left(622 \mathrm{~W} / \mathrm{m}^{2}\right.$ on a pyranometer) on a hot sunny day. The RY 186 dye is one of the main pollutants in textile industries' waste water. This is because it has a stable structure and does not degrade easily. The molecular structure of RY 186 is shown in Figure 3. The as-synthesized ZnO NPs were used to degrade RY 186 in the presence of sunlight. The RY 186 dye solution (10 ppm) was prepared and $20 \mathrm{mg}$ of $\mathrm{ZnO}$ NPs were added into $50 \mathrm{ml}$ of dye solution and stirred in order to reach the adsorption equilibrium. Then, this solution was placed in a dark environment to investigate the adsorption of RY 186 on ZnO NPs surface and absorption spectra were recorded for $50 \mathrm{~min}$. After that, the solution was exposed to direct sunlight and sampling was carried out at fixed time intervals of $10 \mathrm{~min}$ for $130 \mathrm{~min}$. The collected sample was centrifuged and the supernatant was analyzed to record the absorbance spectra of the residual dye.p

\subsection{Characterization techniques}

The absorption spectra of ZnO NPs were recorded by a Shimadzu UV 2600 spectrophotometer. Fourier transformed infrared (FTIR) spectra were recorded on a Bruker Alpha FTIR spectrometer. The field emission scanning electron microscopy (FESEM) analysis was carried out using a Carl Zeiss field emission scanning electron microscope. The energy dispersive X-ray (EDX) analysis was performed on EDX equipment (Oxford Instruments) to analyze the chemical purity of the sample. A Hitachi HF 3300 transmission electron microscope was used to analyze the morphology and particle size of the as-synthesized ZnO NPs.

\section{Results and discussion}

\subsection{UV-vis analysis}

The UV-vis spectrum of the prepared biogenic $\mathrm{ZnO}$ NPs is described in Figure 4. The sharp absorption peak observed 

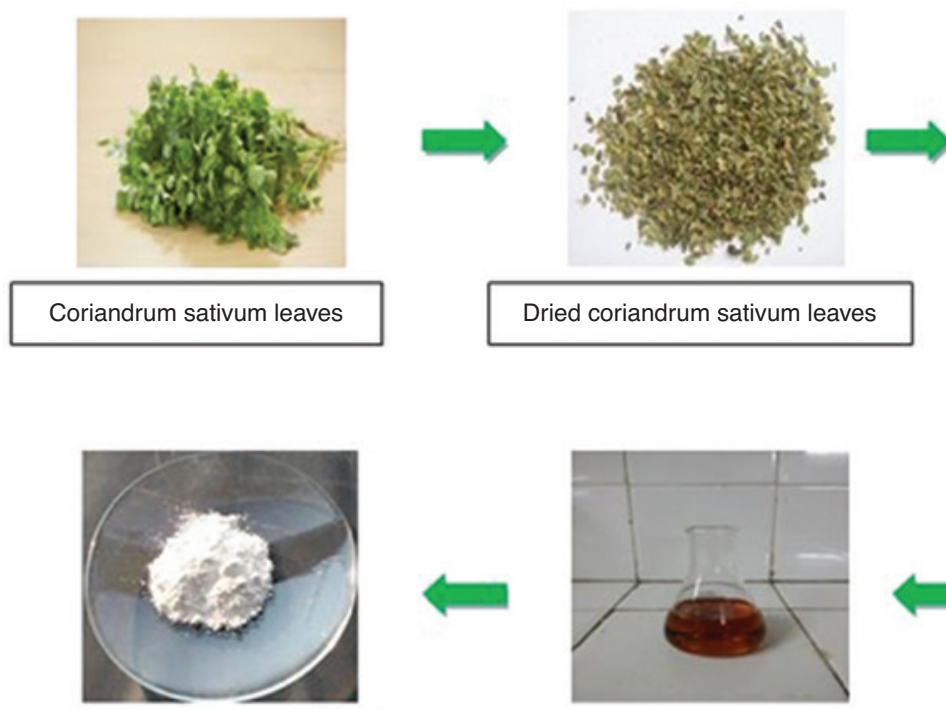

Zinc oxide nanoparticles



Extract with zinc acetate

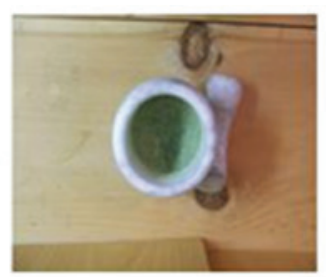

Fine powder of leaves
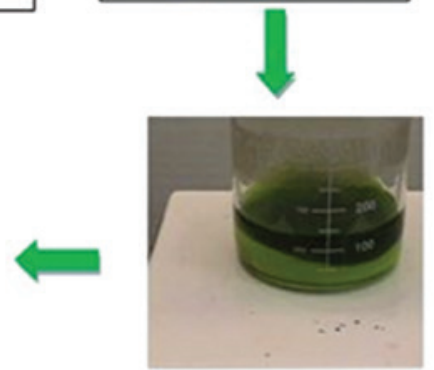

Fine powder in distilled water

Figure 2: Schematic representation of synthesis of $\mathrm{ZnO}$ nanoparticles by Coriandrum sativum leaves.

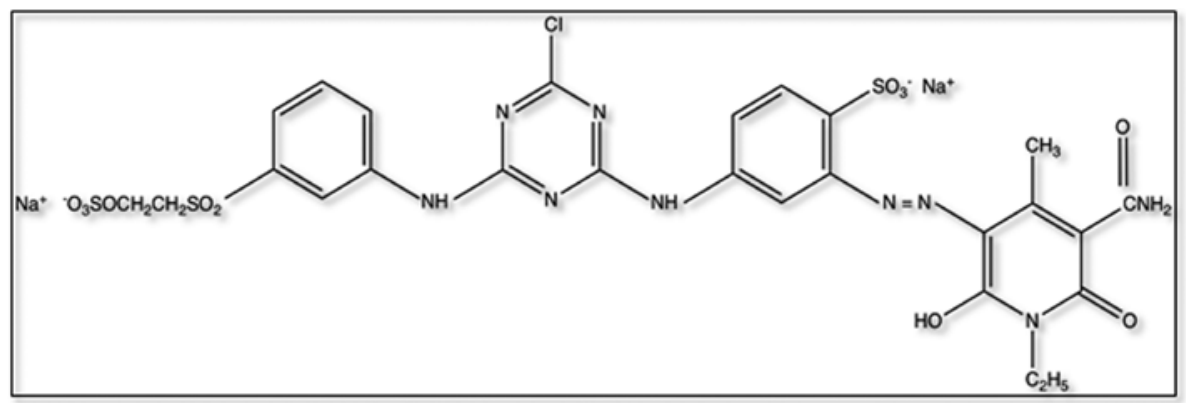

Figure 3: Structure of reactive yellow 186 dye.



Figure 4: UV-vis absorption spectra of $\mathrm{ZnO}$ nanoparticles (NPs). at $364 \mathrm{~nm}$ confirms the formation of ZnO NPs by the autocombustion method. There was a significant blueshift in the excitonic absorption of the prepared biogenic ZnO NPs (364 nm) compared to that of the bulk $\mathrm{ZnO}$ (373 nm) [22, 23]. In addition, there was no other peak observed in the spectrum except for the characteristic peak, which indicates that ZnO NPs prepared using Coriandrum sativum possess high purity. The blueshift in the excitation absorption clearly indicates the quantum confinement property of NPs. In the quantum confinement range, the band gap of the particles increased resulting in the shift of the absorption edge to a lower wavelength, as the particle size decreased. The optical band gap determined from absorption spectra was $3.4 \mathrm{eV}$, which is higher than that of bulk $\mathrm{ZnO}$ (3.32 eV). The band gap of biogenic $\mathrm{ZnO}$ was calculated as: 


$$
\mathrm{E}=\text { h.c } / \lambda_{\text {(absorption) }}=1240 / \lambda_{\text {(absorption) }}
$$

where $\mathrm{h}$ is Planck's constant $\left(6.626 \times 10^{-34} \mathrm{Js}\right), \mathrm{c}$ is the velocity of light $\left(3 \times 10^{8} \mathrm{~ms}^{-1}\right)$ and $\lambda$ is the absorption wavelength of synthesized biogenic $\mathrm{ZnO}$ NPs. $\mathrm{E}=1240 / 364 \mathrm{~nm}=3.4 \mathrm{eV}$.

\subsection{FTIR studies}

The chemical composition of the biogenic ZnO NPs was determined using FTIR spectroscopy and is shown in Figure 5. The FTIR spectrum of prepared ZnO NPs exhibited strong absorption bands at $3692 \mathrm{~cm}^{-1}$ due to O-H stretching vibrations. The peak around $2927 \mathrm{~cm}^{-1}, 2845 \mathrm{~cm}^{-1}$ corresponds to $\mathrm{C}-\mathrm{H}$ stretching. The band at $1648 \mathrm{~cm}^{-1}$ could be associated with $\mathrm{C}=\mathrm{O}$ stretching. The peak near $1400 \mathrm{~cm}^{-1}$ and $1012 \mathrm{~cm}^{-1}$ corresponds to the asymmetry and symmetry vibration of the $-\mathrm{COOH}$ group and $\mathrm{C}-\mathrm{N}$ stretch, respectively. The formation of $\mathrm{ZnO}$ NPs was confirmed by the peak at $782 \mathrm{~cm}^{-1}$ which corresponds to the $\mathrm{ZnO}$ stretching vibration [24].

\subsection{X-ray diffraction analysis}

The X-ray diffraction (XRD) pattern shown in Figure 6 confirms the formation of $\mathrm{ZnO}$ NPs with a wurtzite structure. The strong intensity of the diffraction peaks exhibits the crystalline nature of the as-synthesized ZnO NPs. The diffraction peaks at $31.77^{\circ}, 34.43^{\circ}, 36.21^{\circ}, 47.71^{\circ}, 56.71^{\circ}$, $63.00^{\circ}, 66.49^{\circ}, 68.09^{\circ}$ and $69.16^{\circ}$ indicate (1 00 ) $),\left(\begin{array}{lll}0 & 0 & 2\end{array}\right),(1$

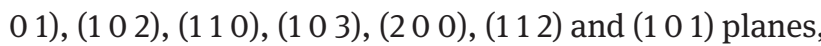
respectively, of $\mathrm{ZnO}$ resembling the standard data of the JCPDS Card number: 36-1451. The average crystalline size

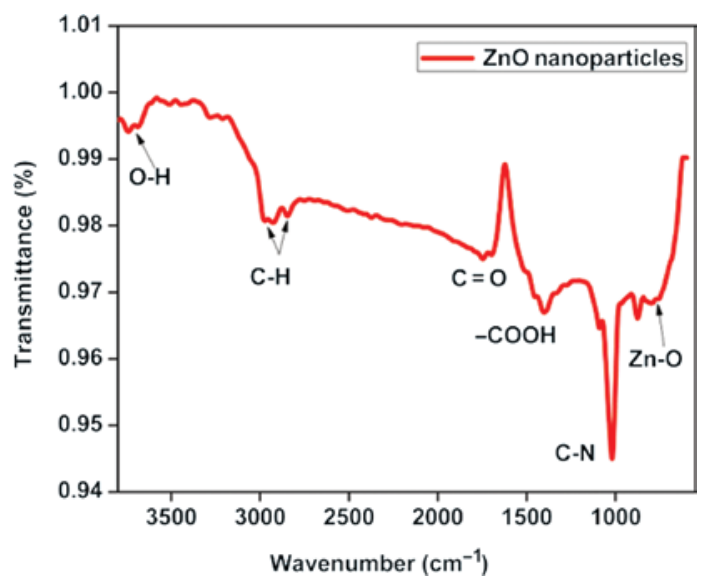

Figure 5: Fourier transform infrared (FTIR) spectra of biogenic ZnO nanoparticles (NPs).



Figure 6: X-ray diffraction (XRD) pattern of the synthesized biogenic ZnO nanoparticles (NPs).

of the prepared ZnO NPs was determined using DebyeScherrer's formula [25]:

$$
\mathrm{D}=\frac{0.9 \lambda}{\beta \cos \theta}
$$

where $\lambda$ is the $\mathrm{X}$-ray wavelength, $\theta$ is the Bragg angle and $\beta$ is the full width at half maximum (FWHM). The calculated crystalline size of the synthesized ZnO NPs was $34 \mathrm{~nm}$.

\subsection{Morphological studies}

Transmission electron microscopy (TEM) analysis represented spherical ZnO NPs with an average particle size of $24 \mathrm{~nm}$, as displayed in Figure 7A and B, which is in accordance with the XRD results. The FESEM images show the external morphology of ZnO NPs as having a spherical shape and small aggregates, as shown in Figure $8 \mathrm{~A}$ and B. The EDX study of ZnO NPs prepared using Coriandrum sativum leaves extract was carried out to determine their elemental composition as presented in Figure 8C. From the EDX spectra, it can be seen that the sharp peaks for $\mathrm{Zn}$ and $\mathrm{O}$ are $80.56 \%$ and $19.44 \%$, respectively, which indicates the formation of $\mathrm{ZnO}$ NPs.

\subsection{Photocatalytic activity}

In UV-vis spectroscopy, the characteristic peak of RY 186 dye is exhibited at $418 \mathrm{~nm}$. As the irradiation time increases, the intensity of the characteristic peak decreases, indicating that complete degradation of the dye by ZnO NPs 




Figure 7: Transmission electron microscopy (TEM) images of ZnO nanoparticles (NPs).

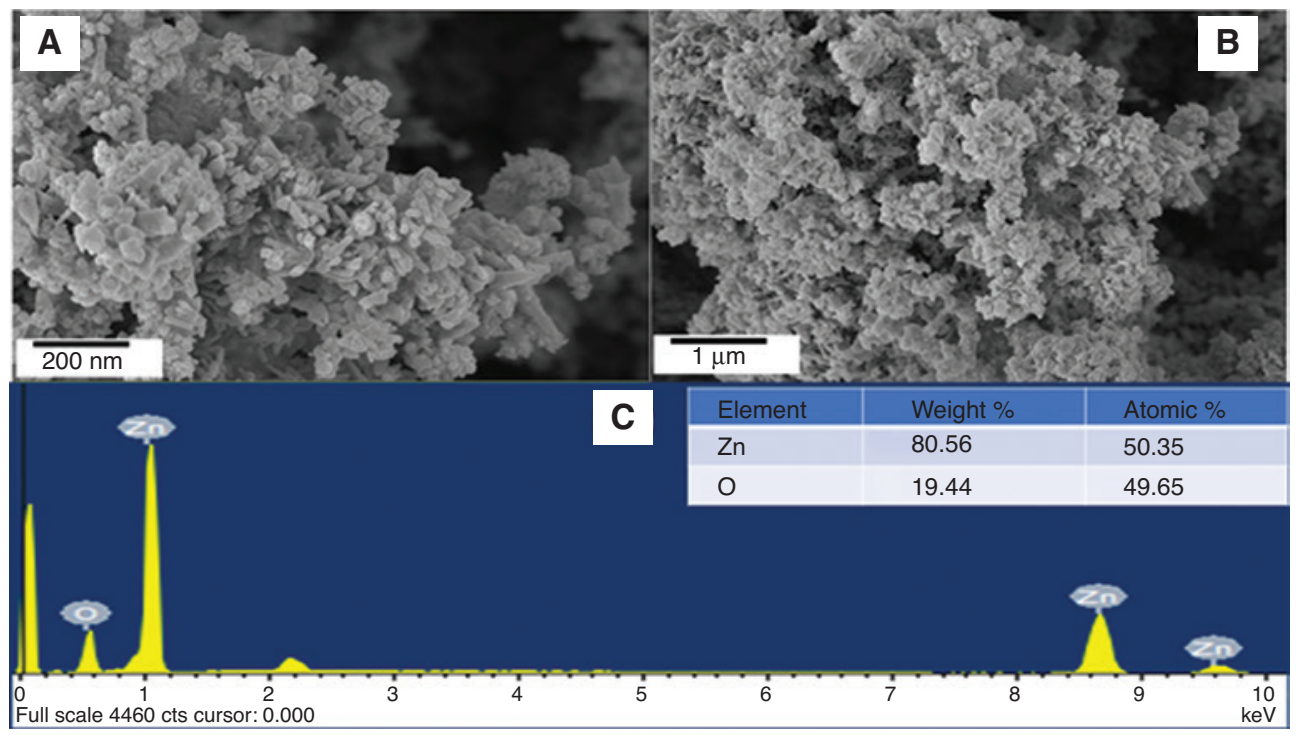

Figure 8: (A) (B) Field emission scanning electron microscopy (FESEM) images, and (C) energy dispersive X-ray (EDX) pattern of ZnO nanoparticles (NPs).

occurred in $2 \mathrm{~h} 10 \mathrm{~min}$, as shown in Figure 9. The dye degradation mechanism was explained by the pseudo-first order rate law [26]:

$$
\ln \left(\mathrm{A} / \mathrm{A}_{0}\right)=-\mathrm{kt}
$$

where $A_{0}$ refers to the absorbance at time $t=0$, A refers to the absorbance at different time intervals during the reaction and $\mathrm{k}$ is the rate constant. After complete degradation of the dye, the rate constant was calculated to be $0.0019 \mathrm{~min}^{-1}$ as depicted in Figure 10. The degradation efficiency was determined from absorption using Lambert Beer's Law:

$$
\mathrm{R}=\left(\mathrm{A}_{0}-\mathrm{A}\right) / \mathrm{A}_{0} \times 100
$$

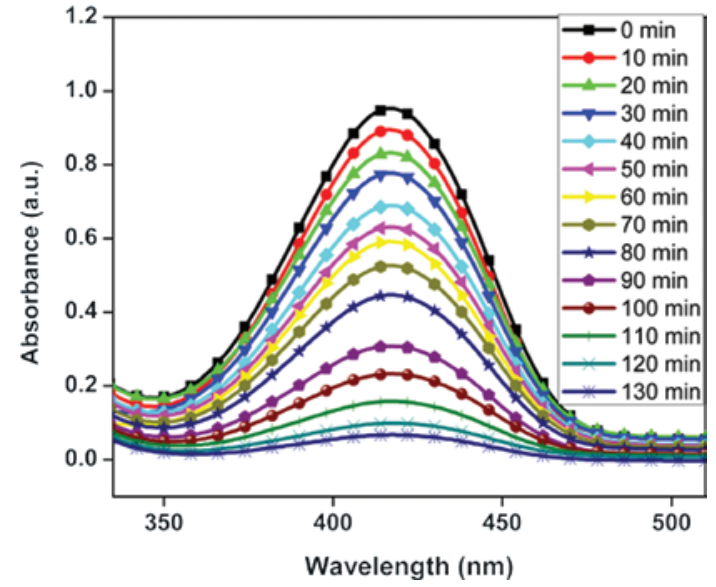

Figure 9: Photocatalytic degradation of reactive yellow 186 dye by $\mathrm{ZnO}$ nanoparticles (NPs). 


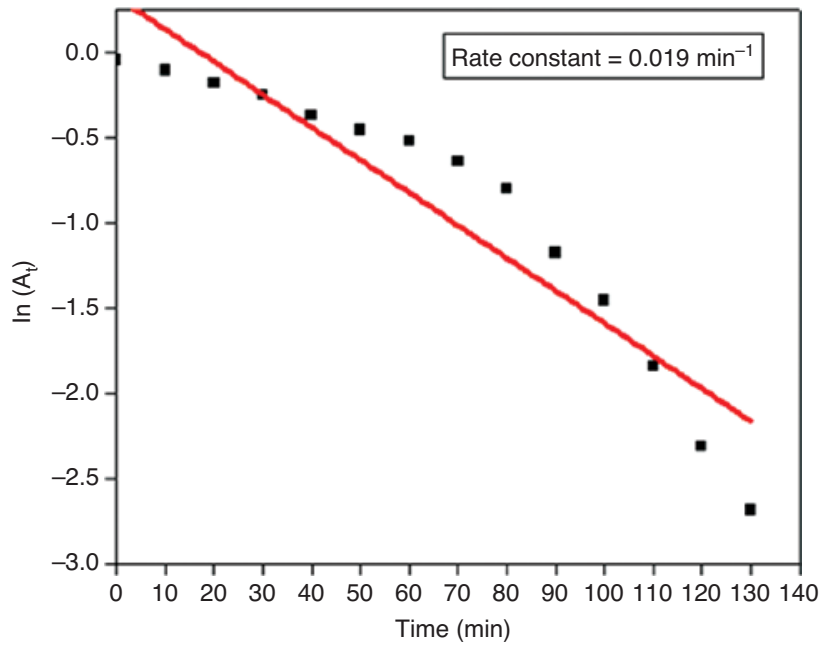

Figure 10: Schematic illustrates the first order kinetic fit for reactive yellow 186 dye.

where $\mathrm{A}_{0}$ and $\mathrm{A}$ correspond to the absorbance of the dye at time $t=0$ and $t$, respectively. The degradation efficiency of RY 186 dye by ZnO NPs was observed to be $93.38 \%$.

The mechanism of dye degradation under sunlight is supposed to be the result of the generation of electron-hole pairs in ZnO NPs, which are very active on the surface. The free holes and electrons moving in valance bands oxidize $\mathrm{H}_{2} \mathrm{O}$ and $\mathrm{O}_{2}$ molecules and generate $\mathrm{OH}^{-}$radicals and $\mathrm{O}^{2-}$ ions, respectively. The highly reactive $\mathrm{OH}^{-}$radicals are responsible for degradation of the RY 186 dye adsorbed on to the surface of ZnO NPs [4, 27]. Due to blueshift and a wide band gap of biogenic ZnO NPs, the overall absorption of sunlight irradiation will be enhanced, which leads to higher degradation efficiency. The proposed mechanism is described in Figure 11.

To investigate the effectiveness of the photocatalyst, control experiments in the dark were further carried out. Insignificant decline in the dye absorbance was observed in the dark period, which suggested negligible adsorption or degradation of the dye in the absence of sunlight (Figure 12). In contrast, under direct sunlight, RY 186 was almost completely degraded over 160 min of experimental duration. These trials evidently established that dye degradation was driven by a photocatalytic process.

In the next step, the reusability of the photocatalyst was investigated. For this purpose, dye treated ZnO NPs were washed four times with water to eradicate the residual dye molecules from their surface. Again, the NPs were suspended in water and their photocatalytic potential for degradation of RY 186 dye was tested. The percentage degradation of RY 186 dye over three successive experiments was calculated to be $93.3 \%, 92.5 \%$, and $90.5 \%$, respectively (Figure 13). These observations demonstrated negligible variation in the photocatalytic potential of the ZnO NPs. Also, XRD of the ZnO NPs after three rounds of photocatalytic degradation experiments was carried out to measure the photostability of the ZnO NPs. As shown in Figure 14, except for a minor decline in peak intensities,

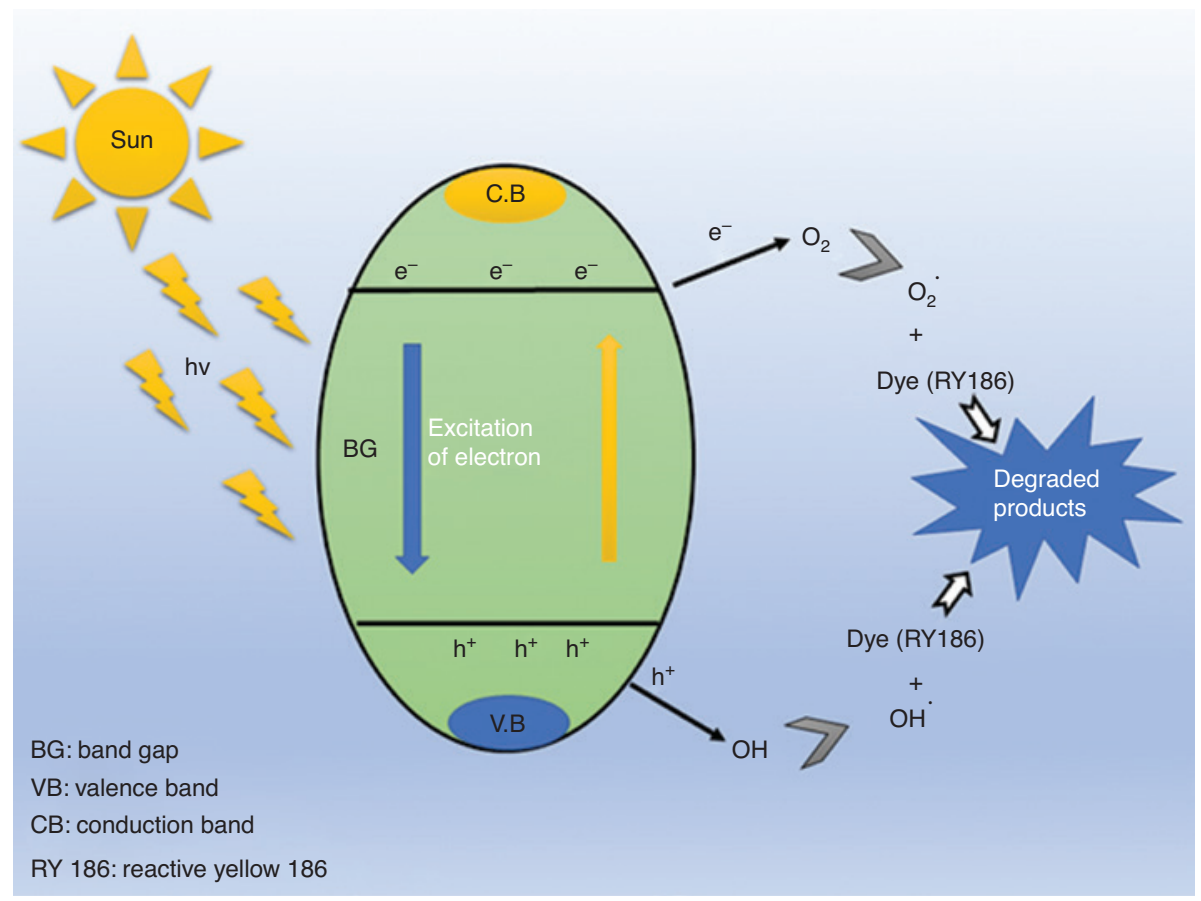

Figure 11: Schematic shows the mechanism of dye degradation by $\mathrm{ZnO}$ nanoparticles (NPs) in direct sunlight. 


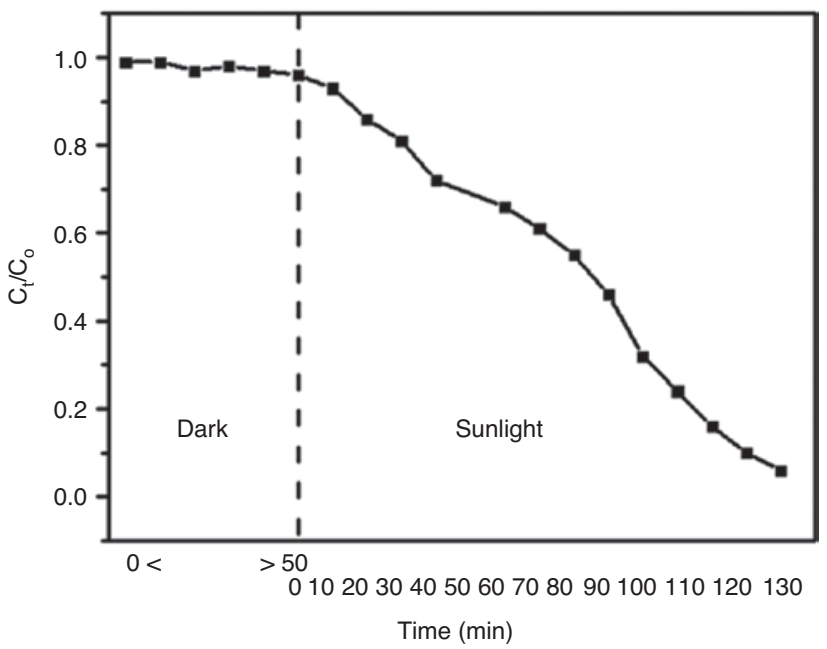

Figure 12: Schematic shows the normalized concentration of dye in dark and sunlight environment.

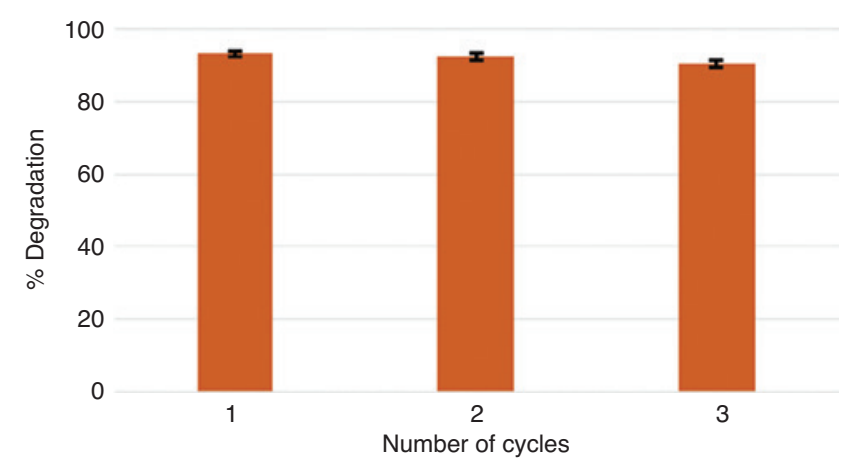

Figure 13: Reusability of $\mathrm{ZnO}$ nanoparticles (NPs) in three successive runs.



Figure 14: X-ray diffraction (XRD) pattern of recyclable ZnO nanoparticles (NPs).




no variation in the powder XRD profile of the ZnO NPs was observed. Hence the NPs showed significant photostability and reusability for photocatalytic degradation of RY 186 dye.

Finally, we compared our study with previous available work as described in Table 1. It was observed that most of the studies employed the chemical methods for synthesis of ZnO NPs, which are noxious, toxic and expensive. Also, lab dyes (methylene blue, Congo red) were used, which are more easily degraded than the industrial dyes (reactive yellow 186). Moreover, in some studies, they prepared the composites, which makes the process complex. In a number of studies, artificial light sources were used, which are expensive and difficult to handle. Likewise, some studies use the adsorption process, which will convert the pollutants from one form to another and not complete mineralization of the pollutants. For instance, in this work a simple, cost-effective, eco-friendly method was used to synthesize the ZnO NPs, which shows the prodigious potential for photocatalytic degradation of industrial dye (RY 186) in direct sunlight environment. Thus, our study provides new insights into green approaches for the synthesis of NPs and their utilization as green photocatalysts for the removal of noxious industrial effluents.

\section{Conclusion}

The present study reports a simple, cost-effective and environmentally friendly synthesis of ZnO NPs using Coriandrum sativum leaf extract and zinc acetate. Various characterization techniques such as UV-vis, FTIR spectroscopy, XRD, TEM, FESEM and EDX analysis confirmed the formation of ZnO NPs. The synthesized biogenic ZnO NPs exhibited a UV-vis absorption peak at $364 \mathrm{~nm}$ and FTIR spectra showed a peak at $782 \mathrm{~nm}^{-1}$ which corresponds to the $\mathrm{ZnO}$ stretching vibration. The FESEM and TEM images revealed a spherical shape of NPs and a particle size of $24 \mathrm{~nm}$, respectively. The estimated optical band gap of $\mathrm{ZnO}$ was found to be $3.63 \mathrm{eV}$, which also clearly indicates the presence of blueshift. The UV absorption results also show an increase in the optical band gap, which further enhances the photocatalytic degradation potential of $\mathrm{ZnO}$ NPs. The synthesized ZnO NPs show a huge potential for photocatalytic degradation of RY 186 with $93.38 \%$ efficiency. Moreover, the synthesized biogenic ZnO NPs show excellent photostability even after being reused three times. Thus, a cost-effective, eco-friendly method was developed for the synthesis of biogenic and wide band gap $\mathrm{ZnO}$ NPs, which can be utilized for waste water treatment.
Acknowledgements: The authors are thankful to the ViceChancellor of SGGSW University for providing the necessary laboratory facilities.

Conflict of interest statement: The authors declare that there are no conflicts of interest regarding the publication of this manuscript.

\section{References}

[1] Wang ZL. J. Phys. Condens. Matter 2004, 16, R829-R858.

[2] Moezzi A, McDonagh AM, Cortie MB. Chem. Eng. J. 2012, 185-186, 1-22.

[3] Sangeetha G, Rajeshwari S, Venckatesh R. Mater. Res. Bull. 2011, 46, 2560-2566.

[4] Wahab R, Khan F, Lutfullah, Singh RB, Kaushik NK, Ahmad J, Siddiqui MA, Saquib Q, Ali BA, Khan ST, Musarrat J, Musarrat AA. Phys. E 2015, 69, 101-108.

[5] Li D, Haneda H. Chemosphere 2003, 51, 129-137.

[6] Pearce Cl, Lloyd JR, Guthrie JT. Dyes Pigm. 2003, 58, 179-196.

[7] Robinson T, McMullan G, Marchant R, Nigam P. Bioresour. Technol. 2001, 77, 247-255.

[8] Djurišić AB, Leung YH, Ching Ng AM. Mater. Horizons 2014, 1, 400.

[9] Mills A, Le Hunte S. J. Photochem. Photobiol., A 1997, 108, $1-35$.

[10] Maryanti E, Damayanti D, Gustian I, Yudha SS. Mater. Lett. 2014, 118, 96-98.

[11] Zak AK, Abrishami ME, Majid WHA, Yousefi R, Hosseini SM. Ceram. Int. 2011, 37, 393-398.

[12] Jamal RK, Hameed MA, Adem KA. Mater. Lett. 2014, 132, 31-33.

[13] Zak AK, Majid WHA, Wang HZ, Yousefi R, Moradi Golsheikh A, Ren ZF. Ultrason. Sonochem. 2013, 20, 395-400.

[14] Ao W, Li J, Yang, H, Zeng X, Ma X. Powder Technol. 2006, 168, 148-151.

[15] Salavati-Niasari M, Davar F, Khansari A. J. Alloys Compd. 2011, 509, 61-65.

[16] Iravani S. Green Chem. 2011, 13, 2638.

[17] Nath D, Banerjee P. Environ. Toxicol. Pharmacol. 2013, 36, 997-1014.

[18] Ramesh P, Rajendran A, Meenakshisundaram M. J. Nanosci. Nanotechnol. 2014, 2, 41-45.

[19] Mahendiran D, Subash G, Arumai Selvan D, Rehana D, Senthil Kumar R, Kalilur Rahiman A. J. Bionanosci. 2017, 7, 530-545.

[20] Rajiv P, Rajeshwari S, Venckatesh R. Spectrochim. Acta A Mol. Biomol. Spectrosc. 2013, 112, 384-387.

[21] Singh RP, Shukla VK, Yadav RS, Sharma PK, Singh PK, Pandey AC. Adv. Mater. Lett. 2011, 2, 313-317.

[22] Xin-Hua L, Jia-Yue X, Min J, Hui S, Xiao-Min L. Chin. Phys. Lett. 2006, 23, 3356.

[23] Debanath MK, Karmakar S. Mater. Lett. 2013, 111, 116-119.

[24] Music S, Popovic S, Maljkovic M, Dragcevic E. J. Alloys Compd. 2002, 347, 324-332.

[25] Patterson AL. Phys. Rev. 1939, 56, 978-982.

[26] Nitoi I, Oncescu T, Oancea P. J. Ind. Eng. Chem. 2013, 19, 305-309. 
[27] Raza W, Haque MM, Muneer M. Appl. Surf. Sci. 2014, 322, 215-224.

[28] Singh G, Singh J, Jolly SS, Rawat R, Kukkar D, Kumar S, Basu S, Rawat M. J. Mater. Sci. Mater. Electron. 2018, 29, 7364-7371.

[29] Dhanavel S, Nivethaa EAK, Narayanan V, Stephen A. Int. J. ChemTech Res. 2014, 6, 1880-1882.
[30] Bagheri M, Mahjoub AR, Mehri B. RSC Adv. 2014, 4, 21757-21764.

[31] Sharma M, Hazra S, Basu S. J. Colloid Interface Sci. 2017, 504, 669-679.

[32] Ali M, Idris M, Quayum M. J. Nanostruct. Chem. 2013, 3, 36.

[33] Vidya C, Chandra Prabha MN, Antony Raj MAL. Environ. Nanotechnol. Monit. Manag. 2016, 6, 134-138. 